\title{
Laboratory Assessment of Host Plant Selection of the Brown Marmorated Stink Bug (Halyomorpha halys)
}

\author{
Beatrice Nuck Dingha*, Sushil Nyaupane, Louis Ernest Jackai \\ Department of Natural Resources and Environmental Design, North Carolina A and T State University, Greensboro, USA
}

Email address:

bndingha@ncat.edu (B. N. Dingha)

${ }^{*}$ Corresponding author

To cite this article:

Beatrice Nuck Dingha, Sushil Nyaupane, Louis Ernest Jackai. Laboratory Assessment of Host Plant Selection of the Brown Marmorated Stink Bug (Halyomorpha halys). American Journal of Entomology. Vol. 4, No. 2, 2020, pp. 26-34. doi: 10.11648/j.aje.20200402.11

Received: June 22, 2020; Accepted: July 16, 2020; Published: August 10, 2020

\begin{abstract}
The behavioral response of brown marmorated stink bug, Halyomorpha halys (Hemiptera: Pentatomidae), to various phenological growth stages (leaf, flower and pod/seed/kernel) of six host plants (soybean, corn, sunflower, Early Scarlet and Mississippi Silver cowpeas, and princess tree leaf) was investigated in a Dual Choice Arena Test (DCAT) and Multiple Choice Arena Test (MCAT). In DCAT, each experiment consisted of one of the growth stages of each plant with princess tree leaf as the standard. In MCAT, two experiments were conducted using: (1) similar growth stages from the different plants and (2) different growth stages from the same plant. Halyomorpha halys distribution was observed at time intervals up to 24 hours after insects were released. Results from MCAT and Preference Index (PI) from the DCAT indicated that more $H$. halys were recorded on princess tree leaves. Mississippi Silver $(\mathrm{PI}=1.59 \pm 0.05)$ and Early Scarlet $(\mathrm{PI}=1.49 \pm 0.08)$ flowers were preferred over princess tree leaves and other flowers $(\mathrm{PI} \leq 1)$. Sunflower seed was the least preferred $(\mathrm{PI}=0.43 \pm 0.18)$ among similar growth stages. Overall, the reproductive stages were preferred over the vegetative stage. Our findings can be useful in developing management programs such as trap cropping for this pest.
\end{abstract}

Keywords: Halyomorpha halys, Brown Marmorated Stink Bug, BMSB, Host Plant Selection, Preference Index, Vegetable Crops

\section{Introduction}

Halyomorpha halys Stal (Hemiptera: Pentatomidae), commonly referred to as the brown marmorated stink bug (BMSB), is an invasive pentatomid species in the United States. Native to Asia, it was first detected in the United States in the mid-1990's in Allentown, Pennsylvania. More than two decades later, $H$. halys population size and distribution have steadily grown and spread widely. Currently, H. halys has been detected in more than 46 states and is considered a severe agricultural and residential pest [1]. It has been hypothesized that with the increase in global temperature, invasive species such as $H$. halys will increase in numbers and have the potential of expanding to new areas [2]. The first report of $H$. halys in Canada was in 2010, in Ontario and Québec [3]. In Europe, the first established population was detected and reported in Zurich, Switzerland, in 2007 [4, 5]. Since then it has spread to multiple European countries including: France [6], Germany [7], Italy [8], Greece [9], Austria [10], Hungary
[11], and Romania [12].

Halyomorpha halys is highly polyphagous and has been documented to feed on a wide range of plant species worldwide causing severe damage $[1,13]$. Generally, phytophagous insects prefer to feed on host plants that provide optimal nutritional quality for reproduction and development [14]. The greatest agricultural damage by $H$. halys has been recorded on vegetable and fruit crops. According to the United States Apple Association [15], H. halys caused \$37 million worth of injury to apples in 2010. In addition, many mid-Atlantic growers reported major yield losses in soybean, sweet corn, peaches, peppers and tomatoes due to this pest [1, 16]. Presently, pesticides are the main strategy for the management of $H$. halys. The lack of effective control measures has prompted many growers to rely on frequent applications of insecticides to increase $H$. halys kill; as much as a 5 -fold increase in insecticide applications has been reported [16]. This practice also kills beneficial insects leading to the resurgence of secondary pests as well as the 
development of insect resistance. Considering the damage and losses incurred by agricultural producers and dangers of frequent insecticide use to humans and the environment, there is need to develop more effective and less risky control methods. The objective of this study was to investigate selective behavior of $H$. halys among host plants and its preference of the different phenological growth stages. The goal of this study was to eventually use the host preference information to develop trap cropping technology $[17,18]$ as an alternative to sole reliance on chemical control. Trap cropping is accomplished by planting a preferred plant variety or species to intercept or divert pests from the main crop. It relies on the pest having a higher preference for specific phenological stage or stages of the preferred plant [19].

\section{Materials and Methods}

\subsection{Experimental Insects and Test Plants}

Adult $H$. halys were obtained from a culture established at the Integrated Pest Management (IPM) Laboratory at North Carolina A\&T State University, Greensboro, North Carolina. Insects were reared at $26 \pm 2{ }^{\circ} \mathrm{C}, 70 \% \mathrm{RH}$ and $16: 8$ (L: D, H) and fed a mixture of food substrates including organic green beans, Phaseolus vulgaris; tomatoes, Solanum lycopersicon; carrot, Daucus carota; sweet corn, Zea mays and leaves of princess tree, Paulownia tomentosa. Moist cotton balls were placed in cages to provide water and maintain humidity. Food was replenished three times within a week or as needed and water daily [20]. During the summer months, adult brown marmorated stink bugs were collected from contiguous integrated pest management experimental plots and princess tree at the North Carolina A\&T State University research farm in Greensboro, North Carolina $\left(36.0586243^{\circ} \mathrm{N}, 79.7358932^{\circ}\right.$ $\mathrm{W})$ and added to the laboratory culture to reduce any inbreeding. Insects were hand collected and placed in $500 \mathrm{ml}$ transparent polypropylene containers with a partial mesh screen lid (BioServ, Frenchtown, New Jersey, USA) containing moist cotton balls and transported to the IPM laboratory where they were transferred to the main culture. During transfer of insects into plastic containers or cages, soft artist's paint brush (Camel Hair, number 2; BioQuip, California, USA) were used to carefully handle the insects to avoid injury or death.

Six host plants of $H$. halys were evaluated, these included soybean, Glycine max (var. 95M82); cowpea, Vigna unguiculata (var. Early Scarlet [ES]) and (var. Mississippi Silver [MS]); corn, Zea mays (var. Trucker's Favorite); sunflower, Helianthus annus (var. Zohar F1OG) and princess tree, Paulownia tomentosa. Plant species were selected in part based on published literature of $H$. halys preference. For example, sunflower was reported to attract significantly more H. halys than okra, Abelmoschus esculentus Moench; admiral pea, Pisum sativum and pearl millet, Pennisetum glaucum [21]. Sweet corn had significant higher abundance of brown marmorated stink bug compared to green bean, eggplant, Solanum Melongena and tomato [22]. Substantial high populations of brown marmorated stink bug were recorded on soybean $[23,24]$ and on Mississippi Silver and Early Scarlet cowpeas $[20,25]$. Also, selection of host plants was based from previous observations of $H$. halys presence on these plants. For example, in the mid-Atlantic region, $H$. halys were first observed on princess tree leaves in June [26]. Similarly, at the NC A\&T State University research farm [20] observed adult $H$. halys that emerged from overwintering sites during the months of April and May preferred the princess tree leaves. Plants were grown at the NC A \& T State university research farm and different plant parts (leaves, flowers and kernels/seeds/pods) were collected as needed for experiments. Mature cowpea and soybean pods were harvested at the R6 stage (50\% of pods with fully developed seeds), corn (R3 \& R4 milk stage), sunflower (stage 8) with a sharp knife and placed in brown paper bags and transported to the laboratory. All plants used in the tests were cleaned with a solution containing $0.5 \%$ bleach to remove any contaminants and air-dried prior to being used. We had established that the bleach solution did not affect feeding by the insect [20].

\subsection{Dual Choice Arena Test (DCAT)}

A dual choice bioassay was performed with princess tree leaf (PT) as the standard. This leaf was used as the standard because after $H$. halys emerges from their overwintering sites, they were only observed on princess tree leaves [20] which is usually the main plant present. Three DCAT (PT-X) experiments were set up, where $\mathrm{X}$ consist of one of the three phenological growth stages (leaf, flower and corn $\mathrm{kernel} / \mathrm{seed} /$ pod) of each host plant tested against princess tree leaf (PT). Leaf discs $2.5 \mathrm{~cm}$ (in diameter) cut with a cork borer, mature pods $5 \mathrm{~cm}$ long, and flowers were used. For sunflower and corn, 10 seeds and corn cob with 15 kernels were used respectively. Efforts were made to use plant parts of uniform size and age. A circular plastic container $(20 \times 8 \mathrm{~cm}$; Pioneer Plastic Inc. Dixon, KY, USA) was used as the experimental arena, the bottom of which was covered with moist paper towel to prevent desiccation of the plant material. Whatman filter paper that fits the base of the experimental arena was divided into four equal quadrants each with a $90^{\circ}$ angle at the center was placed on top of the moist paper towel. In the first experimental setup, single leaf discs of a test plant were placed in opposite quadrants and the standard (princess tree) on the remaining two quadrants (in total, four leaf discs for each arena). The second experimental setup consisted of single flower of a test plant placed in opposite quadrants and leaf discs of princess tree on the remaining two quadrants. And the third comprised of single pod/corn kernel/seeds of test plant placed in opposite quadrants and the standard in the remaining two quadrants. Since princess tree was observed to be highly preferred by $H$. halys on the field when other host plants were absent, a no choice test consisting of only leaf discs of princess tree (PT-PT) in all four quadrants was setup as a control. Data from this experimental setup was used to make comparisons between number of $H$. halys recorded in a choice test and a no choice test. To begin an assay, four adult $H$. halys previously starved for 24 hours were gently released 
into the center of the arena with an artist's paint brush (Camel Hair, number 2; BioQuip, California, USA). In both experimental setup (choice and no choice tests), each treatment was replicated 10 times. The experiments were conducted in a Complete Randomized Design (CRD). Data on the total number of insects present on both plant parts of the test plant and that on the standard at 2, 4, 6, 8, 10, 12 and $24 \mathrm{~h}$ after their release was collected and recorded. At the end of the DCAT evaluation, the preference index (PI) of each test plant was determined.

\subsection{Multiple Choice Arena Test (MCAT)-Growth Stage Selection}

Multiple choice tests were conducted to examine $H$. halys selection behavior of plant phenological growth stages (leaf, flower, corn kernel/seed/pod) among test plants. The arena used in these experiments were similar to those described in the previous experiments (DCAT) with slight modification consisting of six lines drawn across the Whatman filter paper for a total of six hexagonal sections each with a $60^{\circ}$ angle at the center.

\subsubsection{Similar Growth Stages from the Different Test Plants}

The bioassay included three treatments (leaf, flower and corn kernel/seed/pod). In each arena, a single leaf disc from each test plant and the standard was placed at the center of each hexagonal section for a total of six leaf discs. This setup was repeated for flowers and corn kernel/seeds/pods of each test plant with leaf disc of the standard. Six previously starved adult $H$. halys were released at the center of the arena and the insects allowed to make a choice. There were nine replications in a Complete Randomized Design (CRD). Data on the number of $H$. halys on each plant part $1,2,3,4,5,6,7,8$ and 24 hours after release of the insects was collected and recorded.

\subsubsection{Different Growth Stages from the Same Test Plant}

In this experiment, there were five treatments (soybean, Early Scarlet, Mississippi Silver, sweet corn and sunflower). For each treatment, the three phenological growth stages were placed at the center in alternate hexagonal sections and replicated nine times. Six previously starved adult BMSBs were released at the center of the arena. Data on the number of $H$. halys on each plant part 1, 2, 3, 4, 5, 6 and 24 hours after release of the insects was collected and recorded.

\subsection{Data Analysis}

Experiments were set up as a Complete Randomized Design (CRD) with each arena as a replicate. The index of comparison in the DCATs is the Preference Index (PI). PI for each test plant relative to the standard was calculated as $\mathrm{PI}=2 * \mathrm{~T} /(\mathrm{T}+\mathrm{C})[21,22]$, Where $\mathrm{T}$ is the number of insects on the test plant and $\mathrm{C}$ the number of insects on the standard plant The PI value ranges from 0 to 2 , with a PI of 1 indicating no preference for either $\mathrm{C}$ or $\mathrm{T}$ plant, a $\mathrm{PI}>1$ indicating preference for $\mathrm{T}$ test plant, and a $\mathrm{PI}<1$ indicating preference for the standard (C). All data were analyzed using one-way Analysis of variance (ANOVA) using the general linear model (GLM procedure SAS version, 9.3, Cary, NC, USA). Repeated measures analysis of variance (ANOVA) was used to compare the distribution of $H$. halys on host plants over time. Treatment means were separated using Tukey's Honest Significant Difference test $(\mathrm{P}<0.05)$.

\section{Results}

\subsection{Dual Choice Arena Test (DCAT)}

From the DCAT, when $H$. halys was given a choice between leaves of princess tree and leaves from the other host plant (MS, ES, soybean, sunflower or corn) using princess tree leaf as the standard and data collected at each time interval $(2,4,6$, $8,10,12$ and $24 \mathrm{~h}$ ), our results show that at each time interval except at 2 hours (Mississippi Silver leaf), the PI was $\leq 1$ for all the leaves of the test plants indicating preference for princess tree leaf by BMSB (Table 1a). At 2 hours after release of BMSB in the test arena, the PI values among the leaves of test plants was not significantly different even though Mississippi Silver with a PI value of $1.7+0.24$ was preferred over princess tree leaf by $H$. halys (Table 1a). At 4 and 8 hours, the PI value of sunflower leaf was 1 indicating no preference for either sunflower or princess tree leaves. Also, at 24 hours, the PI value of Early Scarlet was 1 (Table 1a). At each time interval there were no significant differences among the PI values of the test plant leaves (Table 1a). The mean Preference Index (PI) calculated from the data collected at 2, 4, 6, 8, 10, 12 and 24 hours after the release of BMSB in the test arena, shows that the PI for leaves from the other host plants were $<$ 1 indicating $H$. halys preferred princess tree leaves over the other leaves (Figure 1a). Among the five test plants, there was no significant difference $(\mathrm{F} 4,30=1.9, \mathrm{P}=0.1332)$ in the PI of the leaves (Figure 1a).

For the flowers, the PI values of Mississippi Silver and Early Scarlet flowers were $\geq 1$ at each time interval $(2,4,6,8$, 10,12 ) except after 24 hours (Table 1b), indicating that these flowers were preferred over princess tree leaf at these times. Generally, at each time interval there were significant differences among the PI values of the test plant leaves with Mississippi Silver and Early Scarlet flowers recording higher PI values (Table 1b). The mean PI values of Mississippi Silver (MS) and Early Scarlet (ES) cowpea flowers were $>1$ indicating their flowers were preferred over princess tree leaf while $H$. halys preferred princess tree leaves over flowers of soybean, corn and sunflower (Figure 1b). There was a significant difference $(\mathrm{F} 4,30=45.9, \mathrm{P}=0.0001)$ in the PI values among the flowers of the test plants with Mississippi Silver (MS) and Early Scarlet (MS) highly preferred (PI=1.5) and corn the least preferred $(\mathrm{PI}=0.12)$.

At 2, 4, 6, 8, 10 and 12 hours after the release of $H$. halys in the arena, the PI values were $\geq 1$ for pods of soybean, Mississippi Silver and Early Scarlet and for soybean pod at 24 hours and for sweet corn kernel, the PI values were $\geq 1$ at 4,6 , $8,10$ and 12 hours (Table $1 \mathrm{c})$. These PI values $(\geq 1)$ indicate these test plant parts were preferred over princess tree leaf. For 
each time interval there were significant differences in the PI value among the test plants with pods of Mississippi Silver, Early Scarlet cowpeas and soybean and corn kernel recording higher PI values (Table 1c). The mean PI values indicated that the pods of soybean, Mississippi Silver and Early Scarlet cowpeas, and sweet corn seeds/kernel were preferred over princess tree leaves (Figure 1c). There was a significant difference $(\mathrm{F} 4,30=22.4, \mathrm{P}=0.0001)$ in the PI values among the pods/seeds of the different test plants. Sunflower seed was the least preferred with $\mathrm{PI}=0.5$ indicating that $H$. halys preferred princess tree leaf over sunflower seeds (Figure 1c).

Table 1. Mean ( \pm SE) Preference Index (PI) of H. halys recorded on (a) leaves (b) flowers (c) kernels/seeds/pods of different host plants in a Dual Choice Arena Test (DCAT) at 2, 4, 6, 8, 10, 12 and 24 hours after release.

\begin{tabular}{|c|c|c|c|c|c|c|c|}
\hline \multirow{2}{*}{ Time (h) } & \multicolumn{7}{|l|}{ Mean PI } \\
\hline & 2 & 4 & 6 & 8 & 10 & 12 & 24 \\
\hline \multicolumn{8}{|l|}{ (a) Leaves } \\
\hline Corn & $0.9 \pm 0.31$ & $0.2 \pm 0.15$ & $0.3 \pm 0.23$ & $0.1 \pm 0.14$ & $0.7 \pm 0.32$ & $0.8 \pm 0.28$ & $0.5 \pm 0.24$ \\
\hline ES & $0.7 \pm 0.29$ & $0.6 \pm 0.29$ & $0.4 \pm 0.29$ & $0.2 \pm 0.17$ & $0.3 \pm 0.22$ & $0.0 \pm 0.00$ & $1.0 \pm 0.29$ \\
\hline MS & $1.7 \pm 0.24$ & $0.8 \pm 0.32$ & $0.6 \pm 0.29$ & $0.8 \pm 0.32$ & $0.4 \pm 0.29$ & $0.4 \pm 0.24$ & $0.7 \pm 0.30$ \\
\hline Soybean & $0.8 \pm 0.32$ & $0.5 \pm 0.23$ & $0.9 \pm 0.31$ & $0.8 \pm 0.32$ & $0.6 \pm 0.30$ & $0.5 \pm 0.24$ & $0.7 \pm 0.25$ \\
\hline Sunflower & $0.8 \pm 0.32$ & $1.0 \pm 0.33$ & $0.3 \pm 0.24$ & $1.0 \pm 0.33$ & $0.6 \pm 0.29$ & $0.4 \pm 0.29$ & $0.8 \pm 0.28$ \\
\hline $\mathrm{F}$ & 1.85 & 1.07 & 0.67 & 1.98 & 0.36 & 1.33 & 0.36 \\
\hline $\mathrm{P}$ & 0.1381 & 0.3834 & 0.6185 & 0.1160 & 0.8346 & 0.2755 & 0.8333 \\
\hline \multicolumn{8}{|l|}{ (b) Flower } \\
\hline Corn & $0.0 \pm 0.00_{\mathrm{c}}$ & $0.2 \pm 0.22_{\mathrm{c}}$ & $0.0 \pm 0.00_{\mathrm{d}}$ & $0.2 \pm 0.22_{b}$ & $0.2 \pm 0.12_{b}$ & $0.0 \pm 0.00_{c}$ & $0.2 \pm 0.22$ \\
\hline ES & $1.3 \pm 0.32_{\mathrm{ba}}$ & $1.1 \pm 0.27_{\mathrm{ba}}$ & $1.1 \pm 0.21_{\mathrm{ba}}$ & $1.4 \pm 0.29 \mathrm{a}$ & $1.4 \pm 0.29 \mathrm{a}$ & $1.3 \pm 0.29 \mathrm{a}$ & $0.9 \pm 0.27$ \\
\hline MS & $1.4 \pm 0.28_{\mathrm{a}}$ & $1.7 \pm 0.15_{\mathrm{a}}$ & $1.4 \pm 0.25_{\mathrm{a}}$ & $1.4 \pm 0.16_{\mathrm{a}}$ & $1.5 \pm 0.24_{\mathrm{a}}$ & $1.5 \pm 0.25_{\mathrm{a}}$ & $0.6 \pm 0.23$ \\
\hline Soybean & $0.5 \pm 0.23_{\mathrm{bc}}$ & $0.4 \pm 0.24_{c}$ & $0.7 \pm 0.28_{\mathrm{bc}}$ & $0.6 \pm 0.29_{b}$ & $0.6 \pm 0.29 \mathrm{~b}$ & $0.8 \pm 0.26_{\mathrm{ba}}$ & $0.4 \pm 0.23$ \\
\hline Sunflower & $0.9 \pm 0.35_{\mathrm{ba}}$ & $0.9 \pm 0.31_{\mathrm{bc}}$ & $0.2 \pm 0.22_{\mathrm{dc}}$ & $0.4 \pm 0.29 \mathrm{~b}$ & $0.3 \pm 0.24_{b}$ & $0.5 \pm 0.23_{\mathrm{bc}}$ & $0.3 \pm 0.24$ \\
\hline $\mathrm{F}$ & 4.46 & 5.92 & 7.38 & 4.69 & 6.65 & 6.91 & 1.31 \\
\hline $\mathrm{P}$ & 0.0036 & 0.0008 & 0.0001 & 0.0034 & 0.0003 & 0.0003 & 0.2845 \\
\hline \multicolumn{8}{|c|}{ (c) Kernel/Seeds/pods } \\
\hline Corn & $0.4 \pm 0.29 \mathrm{~b}$ & $1.2 \pm 0.32_{\mathrm{ba}}$ & $1.6 \pm 0.24_{a}$ & $1.3 \pm 0.28_{\mathrm{a}}$ & $1.3 \pm 0.28_{\mathrm{a}}$ & $1.4 \pm 0.29 \mathrm{a}$ & $0.5 \pm 0.24_{\mathrm{bc}}$ \\
\hline ES & $1.1 \pm 0.31_{\mathrm{ba}}$ & $1.1 \pm 0.30_{\mathrm{b}}$ & $1.4 \pm 0.22_{\mathrm{a}}$ & $1.4 \pm 0.23_{\mathrm{a}}$ & $1.1 \pm 0.24_{\mathrm{a}}$ & $1.3 \pm 0.31_{\mathrm{a}}$ & $0.5 \pm 0.23_{\mathrm{bc}}$ \\
\hline MS & $1.2 \pm 0.31_{\mathrm{ba}}$ & $1.1 \pm 0.29_{\mathrm{b}}$ & $1.6 \pm 0.18_{\mathrm{a}}$ & $1.5 \pm 0.25_{\mathrm{a}}$ & $1.2 \pm 0.20_{\mathrm{a}}$ & $1.6 \pm 0.24_{\mathrm{a}}$ & $0.8 \pm 0.29_{\mathrm{ba}}$ \\
\hline Soybean & $1.6 \pm 0.23_{\mathrm{a}}$ & $1.7 \pm 0.12_{\mathrm{a}}$ & $1.7 \pm 0.22_{\mathrm{a}}$ & $1.4 \pm 0.29 \mathrm{a}$ & $1.4 \pm 0.28_{\mathrm{a}}$ & $1.3 \pm 0.28_{\mathrm{a}}$ & $1.3 \pm 0.11_{\mathrm{a}}$ \\
\hline Sunflower & $0.3 \pm 0.24_{b}$ & $0.0 \pm 0.00 \mathrm{c}$ & $0.2 \pm 0.22_{\mathrm{b}}$ & $0.2 \pm 0.22_{\mathrm{b}}$ & $0.3 \pm 0.24_{b}$ & $0.0 \pm 0.00_{\mathrm{b}}$ & $0.0 \pm 0.00_{\mathrm{c}}$ \\
\hline $\mathrm{F}$ & 3.74 & 6.65 & 7.35 & 4.12 & 3.08 & 6.11 & 5.19 \\
\hline $\mathrm{P}$ & 0.0113 & 0.0003 & 0.0002 & 0.0069 & 0.0266 & 0.0006 & 0.0018 \\
\hline
\end{tabular}

Abbreviations: ES=Early Scarlet cowpea; MS=Mississippi Silver cowpea; P=P-value; F=F-value.

$\dagger$ Means followed by the same letter within the same column are not significantly different ( $\mathrm{df}=4,40 ; P>0.05$; Tukey's method).

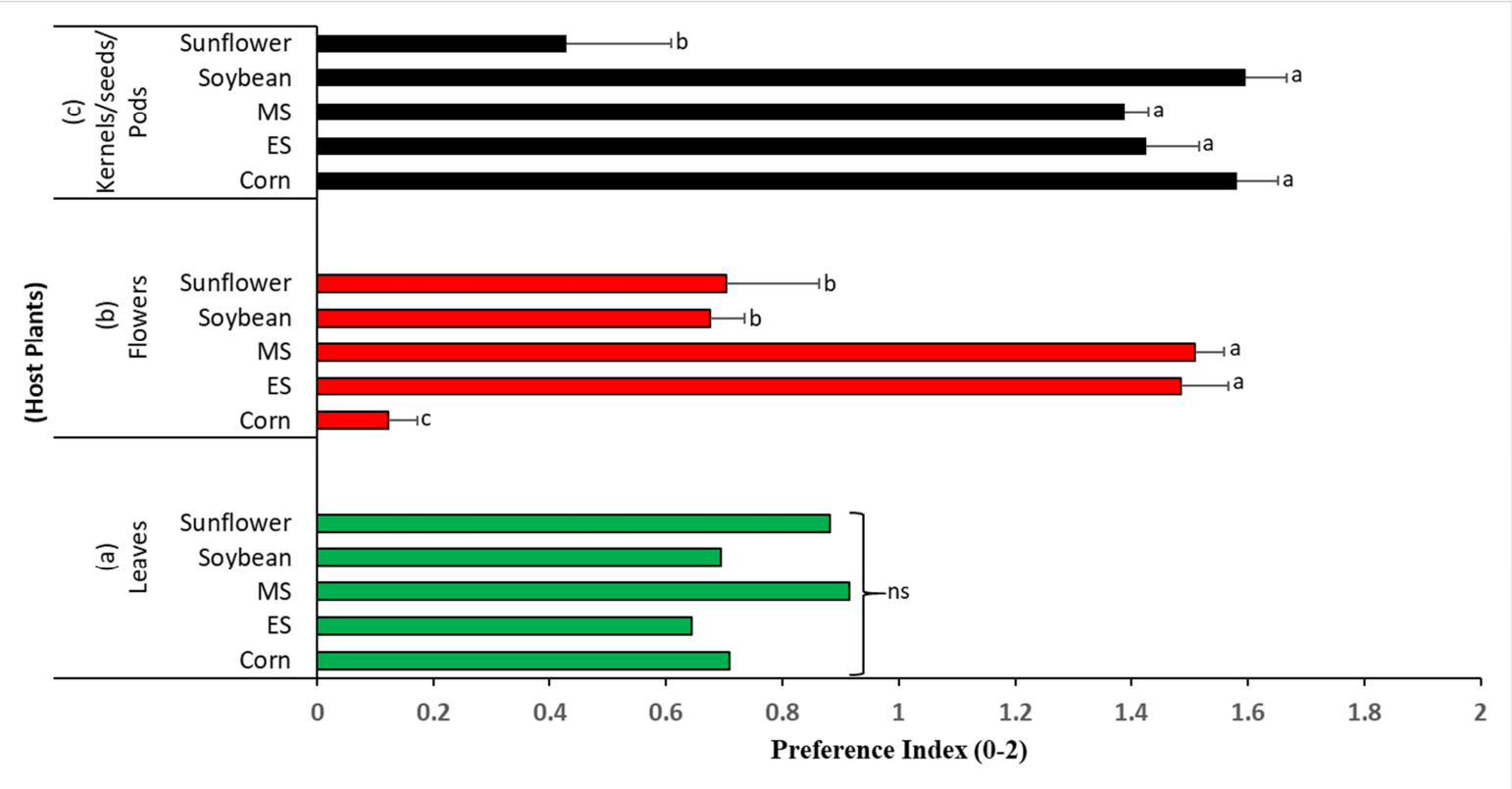

Abbreviations: ES=Early Scarlet cowpea; MS=Mississippi Silver cowpea.

Figure 1. Mean ( \pm SE) Preference Index (PI) of H. halys on (a) leaves (b) flowers and (c) kernels/seeds/pods of different host plants. The PI value ranges from 0 to 2, with a PI of 1 indicating no preference for either princess tree leaves or test plant, a PI $>1$ indicating preference for test plant, and a PI $<1$ indicating preference for the princess tree leaves ( $P>0.05$; Tukey's method). 
The number of $H$. halys recorded on leaves of princess tree in a dual choice bioassay (PT-X, where X is either leaves, flowers or seeds/pods of tested plants; and PT is leaf of princess tree) was compared with that of a no choice test (PT-PT) consisting only of princess tree leaves to determine $H$. halys preference. Our results indicate that at each time interval, the mean number of $H$. halys recorded on princess tree leaves in the no choice test (PT-PT) was higher compared to choice test of leaves of either ES, MS, soybean and corn (Table 2a). This distribution was significantly different at 2, 10, 12 and 24 hours after the release of $H$. halys into the test arena (Table 2a). Similarly, the mean number of $H$. halys recorded in the no choice test (PT-PT) was higher than on a choice test of flowers of either ES, MS, soybean and corn (Table $2 b$ ). This distribution was significantly different at each time interval except at $6 \mathrm{~h}$ (Table 2b). The mean number of $H$. halys recorded on the no choice test (PT-PT) was higher than on the choice test with seeds/pods of either ES, MS, soybean and corn (Table 2c). The distribution was significantly different at each time interval (Table 2c). Overall, the results indicate that in a no choice (PT-PT) setup, the number of $H$. halys recorded on princess tree leaves were higher, however when given a choice (PT-X) of other host plants, the number of $H$. halys recorded on leaf of princess tree decreased (Table $2 \mathrm{a}, \mathrm{b}, \mathrm{c}$ ). From repeated measure analysis, there was no significant effect over time on the number of $H$. halys recorded on test plants.

Table 2. Mean number $( \pm S E)$ of $H$. halys recorded on princess tree leaves in a DCAT when given a choice of (a) leaves (b) flowers (c) kernels/seeds/pods of other host plants (DCAT) and in a no choice test of princess tree leaves (PT-PT) at 2, 4, 6, 8, 10, 12 and 24 hours after release.

\begin{tabular}{|c|c|c|c|c|c|c|c|}
\hline \multirow{2}{*}{ Test plants } & \multicolumn{7}{|c|}{ Time (hours) } \\
\hline & 2 & 4 & 6 & 8 & 10 & 12 & 24 \\
\hline \multicolumn{8}{|l|}{ (a) Leaves } \\
\hline PT-Corn & $0.67 \pm 0.29_{\mathrm{b}}$ & $0.89 \pm 0.35$ & $0.89 \pm 0.35$ & $1.22 \pm 0.36$ & $1.00 \pm 0.33_{\mathrm{b}}$ & $0.89 \pm 0.35_{\mathrm{ba}}$ & $1.33 \pm 0.29 \mathrm{~b}$ \\
\hline PT-ES & $1.00 \pm 0.37 \mathrm{~b}$ & $1.00 \pm 0.41$ & $0.89 \pm 0.39$ & $1.22 \pm 0.40$ & $0.67 \pm 0.37 \mathrm{~b}$ & $1.00 \pm 0.44_{\mathrm{ba}}$ & $1.11 \pm 0.35 \mathrm{~b}$ \\
\hline PT-MS & $0.89 \pm 0.31_{b}$ & $0.56 \pm 0.24$ & $0.67 \pm 0.29$ & $0.56 \pm 0.34$ & $0.67 \pm 0.37 \mathrm{~b}$ & $0.33 \pm 0.17_{\mathrm{b}}$ & $1.00 \pm 0.33_{\mathrm{b}}$ \\
\hline PT-Soybean & $1.00 \pm 0.37_{\mathrm{b}}$ & $1.22 \pm 0.28$ & $1.00 \pm 0.33$ & $1.11 \pm 0.42$ & $1.22 \pm 0.32_{\mathrm{ba}}$ & $1.11 \pm 0.20_{\mathrm{ba}}$ & $1.00 \pm 0.24_{b}$ \\
\hline PT-Sunflower & $0.33 \pm 0.17_{\mathrm{b}}$ & $0.33 \pm 0.24$ & $0.89 \pm 0.26$ & $0.89 \pm 0.39$ & $0.67 \pm 0.24_{b}$ & $0.56 \pm 0.18_{b}$ & $0.89 \pm 0.26_{b}$ \\
\hline PT-PT & $2.11 \pm 0.31_{\mathrm{a}}$ & $1.56 \pm 0.24$ & $1.78 \pm 0.28$ & $2.00 \pm 0.24$ & $2.11 \pm 0.31_{\mathrm{a}}$ & $1.67 \pm 0.33_{\mathrm{a}}$ & $2.22 \pm 0.32_{\mathrm{a}}$ \\
\hline $\mathrm{F}$ & 3.73 & 2.18 & 1.47 & 1.75 & 2.97 & 2.45 & 2.69 \\
\hline $\mathrm{P}$ & 0.0062 & 0.0723 & 0.2180 & 0.1422 & 0.0203 & 0.0467 & 0.0318 \\
\hline \multicolumn{8}{|l|}{ (b) Flowers } \\
\hline PT-Corn & $1.56 \pm 0.41_{\mathrm{a}}$ & $1.78 \pm 0.52_{\mathrm{a}}$ & $1.00 \pm 0.33$ & $1.00 \pm 0.33_{b}$ & $1.44 \pm 0.34_{\mathrm{ba}}$ & $1.56 \pm 0.34_{\mathrm{a}}$ & $1.44 \pm 0.41_{\mathrm{ba}}$ \\
\hline PT-ES & $0.33 \pm 0.17_{\mathrm{b}}$ & $0.67 \pm 0.24_{b c}$ & $1.00 \pm 0.33$ & $0.22 \pm 0.15_{c}$ & $0.33 \pm 0.24_{c}$ & $0.22 \pm 0.15_{b}$ & $0.89 \pm 0.26_{b}$ \\
\hline PT-MS & $0.33 \pm 0.17_{\mathrm{b}}$ & $0.44 \pm 0.24_{c}$ & $0.78 \pm 0.32$ & $0.78 \pm 0.22_{\mathrm{cb}}$ & $0.44 \pm 0.24_{c}$ & $0.67 \pm 0.33_{\mathrm{b}}$ & $1.11 \pm 0.26_{b}$ \\
\hline PT-Sunflower & $0.22 \pm 0.15_{b}$ & $0.78 \pm 0.32_{b c}$ & $0.89 \pm 0.3$ & $0.56 \pm 0.24_{\mathrm{cb}}$ & $1.00 \pm 0.33_{\mathrm{bc}}$ & $0.89 \pm 0.26_{\mathrm{ba}}$ & $0.89 \pm 0.20_{b}$ \\
\hline PT-PT & $2.11 \pm 0.31_{\mathrm{a}}$ & $1.56 \pm 0.24_{\mathrm{ba}}$ & $1.78 \pm 0.28$ & $2.00 \pm 0.24_{\mathrm{a}}$ & $2.11 \pm 0.31_{\mathrm{a}}$ & $1.67 \pm 0.33_{\mathrm{a}}$ & $2.22 \pm 0.32 \mathrm{a}$ \\
\hline $\mathrm{F}$ & 9.35 & 2.80 & 1.27 & 5.55 & 4.65 & 3.69 & 3.37 \\
\hline $\mathrm{P}$ & 0.0001 & 0.0267 & 0.2908 & 0.0004 & 0.0015 & 0.0066 & 0.0109 \\
\hline \multicolumn{8}{|l|}{ (c) Seeds/pods } \\
\hline PT-Corn & $0.22 \pm 0.15_{c}$ & $0.22 \pm 0.15_{\mathrm{c}}$ & $0.22 \pm 0.15_{b}$ & $0.56 \pm 0.24_{b}$ & $0.67 \pm 0.24_{b}$ & $0.56 \pm 0.24_{b}$ & $1.00 \pm 0.33_{\mathrm{b}}$ \\
\hline PT-ES & $0.11 \pm 0.11_{\mathrm{c}}$ & $0.22 \pm 0.15_{c}$ & $0.56 \pm 0.42_{b}$ & $0.78 \pm 0.28_{b}$ & $1.11 \pm 0.35_{\mathrm{b}}$ & $0.78 \pm 0.28_{b}$ & $1.11 \pm 0.31_{b}$ \\
\hline PT-MS & $0.89 \pm 0.39_{b}$ & $1.00 \pm 0.33_{\mathrm{ba}}$ & $0.67 \pm 0.29 \mathrm{~b}$ & $0.67 \pm 0.29_{b}$ & $1.11 \pm 0.26_{\mathrm{b}}$ & $0.67 \pm 0.29_{b}$ & $1.00 \pm 0.33_{\mathrm{b}}$ \\
\hline PT-Soybean & $0.33 \pm 0.17_{\mathrm{cb}}$ & $0.56 \pm 0.18_{\mathrm{bc}}$ & $0.33 \pm 0.17_{\mathrm{b}}$ & $0.44 \pm 0.24_{b}$ & $0.56 \pm 0.24_{b}$ & $0.44 \pm 0.18_{\mathrm{b}}$ & $0.89 \pm 0.11_{b}$ \\
\hline PT-Sunflower & $0.11 \pm 0.11_{\mathrm{c}}$ & $0.56 \pm 0.24_{b c}$ & $0.22 \pm 0.15_{b}$ & $0.33 \pm 0.17_{b}$ & $0.67 \pm 0.24_{b}$ & $0.56 \pm 0.24_{b}$ & $1.44 \pm 0.38_{b}$ \\
\hline PT-PT & $2.11 \pm 0.31_{\mathrm{a}}$ & $1.56 \pm 0.24_{\mathrm{a}}$ & $1.78 \pm 0.28_{\mathrm{a}}$ & $2.00 \pm 0.24_{\mathrm{a}}$ & $2.11 \pm 0.31_{\mathrm{a}}$ & $1.67 \pm 0.33_{\mathrm{a}}$ & $2.22 \pm 0.32_{\mathrm{a}}$ \\
\hline $\mathrm{F}$ & 1.42 & 5.23 & 7.22 & 6.19 & 4.39 & 2.89 & 2.61 \\
\hline $\mathrm{P}$ & 0.0001 & 0.0007 & 0.0001 & 0.0002 & 0.0023 & 0.00231 & 0.0366 \\
\hline
\end{tabular}

Abbreviations: PT-ES=Princess Tree-Early Scarlet cowpea; PT-MS=Princess Tree-Mississippi Silver cowpea; P=P-value; F=F-value.

$\dagger$ Means followed by the same letter within the same column are not significantly different $(\mathrm{df}=5,48$; $\mathrm{P}>0.05$; Tukey's method).

\subsection{Growth Stage Selection-Multiple Choice Arena Test (MCAT)}

\subsubsection{Similar Growth Stages from Different Host Plants}

When $H$. halys was presented with leaves from each test plant including princess tree leaf (PT) in a multiple choice arena test (MCAT), the mean distribution of $H$. halys among the leaves was not significantly different after the first two hours but became significantly different at each hourly time interval after the second hour (Table 3a). During these periods (3-8 $\mathrm{h}$ and $24 \mathrm{~h}$ ) more $H$. halys were recorded on princess tree leaves (PT) compared to leaves from the other host plants
(Table 3a). When $H$. halys was presented with flowers from each test plant including princess tree leaf in a MCAT there was a significant difference in the distribution of $H$. halys among the different flowers and princess tree leaves at various time interval. Generally, flowers of Early Scarlet and Mississippi Silver cowpea had more $H$. halys recorded on them compared to flowers of the other test plants and princess tree leaves (PT) (Table 3b). The mean distribution of $H$. halys among corn kernel/seeds/pods of the different test plants including princess tree leaves (PT) was significantly different at each time interval (Table 3c). During the first three hours 
more H. halys were recorded on soybean pods and corn kernel compared to the others, overall, soybean pod was preferred at every time interval (Table $3 \mathrm{c})$.

Table 3. Mean number $( \pm S E)$ of $H$. halys observed per plant stage (a) leaves, (b) flowers and (c) kernels/seeds/pods with similar growth stages from different host plants in a Multiple Choice Arena Test (MCAT).

\begin{tabular}{|c|c|c|c|c|c|c|c|c|c|}
\hline \multirow{2}{*}{ Test plants } & \multicolumn{9}{|c|}{ Time (hours) } \\
\hline & 1 & 2 & 3 & 4 & 5 & 6 & 7 & 8 & 24 \\
\hline \multicolumn{10}{|l|}{ a) Leaves } \\
\hline Corn & $0.06 \pm 0.06$ & $0.06 \pm 0.06$ & $0.11 \pm 0.08_{b}$ & $0.00 \pm 0.00_{\mathrm{b}}$ & $0.00 \pm 0.00_{b}$ & $0.06 \pm 0.06_{b}$ & $0.00 \pm 0.00_{\mathrm{b}}$ & $0.11 \pm 0.08_{b}$ & $0.17 \pm 0.09_{b}$ \\
\hline ES & $0.06 \pm 0.06$ & $0.00 \pm 0.00$ & $0.06 \pm 0.06_{b}$ & $0.00 \pm 0.00_{\mathrm{b}}$ & $0.00 \pm 0.00_{\mathrm{b}}$ & $0.00 \pm 0.00_{\mathrm{b}}$ & $0.00 \pm 0.00_{\mathrm{b}}$ & $0.00 \pm 0.00_{\mathrm{b}}$ & $0.06 \pm 0.06_{b}$ \\
\hline MS & $0.06 \pm 0.06$ & $0.11 \pm 0.08$ & $0.00 \pm 0.00_{\mathrm{b}}$ & $0.06 \pm 0.06_{b}$ & $0.00 \pm 0.00_{\mathrm{b}}$ & $0.00 \pm 0.00_{\mathrm{b}}$ & $0.00 \pm 0.00_{\mathrm{b}}$ & $0.00 \pm 0.00_{\mathrm{b}}$ & $0.00 \pm 0.00_{\mathrm{b}}$ \\
\hline PT & $0.22 \pm 0.13$ & $0.22 \pm 0.10$ & $0.50 \pm 0.20_{\mathrm{a}}$ & $0.61 \pm 0.16_{a}$ & $0.44 \pm 0.15_{\mathrm{a}}$ & $0.56 \pm 0.17 \mathrm{a}$ & $0.61 \pm 0.24_{\mathrm{a}}$ & $0.56 \pm 0.20_{\mathrm{a}}$ & $0.56 \pm 0.18_{\mathrm{a}}$ \\
\hline Soybean & $0.00 \pm 0.00$ & $0.06 \pm 0.06$ & $0.00 \pm 0.00_{\mathrm{b}}$ & $0.00 \pm 0.00_{b}$ & $0.00 \pm 0.00_{\mathrm{b}}$ & $0.00 \pm 0.00_{\mathrm{b}}$ & $0.00 \pm 0.00_{\mathrm{b}}$ & $0.00 \pm 0.00_{b}$ & $0.06 \pm 0.06_{b}$ \\
\hline $\mathrm{F}$ & 1.11 & 2.05 & 4.62 & 10.57 & 5.47 & 7.80 & 4.30 & 4.11 & 4.56 \\
\hline $\mathrm{P}$ & 0.3612 & 0.0773 & 0.0008 & 0.0001 & 0.0002 & 0.0001 & 0.0014 & 0.0019 & 0.0009 \\
\hline \multicolumn{10}{|l|}{ b) Flowers } \\
\hline Corn & $0.00 \pm 0.00_{\mathrm{c}}$ & $0.06 \pm 0.06_{b}$ & $0.06 \pm 0.06_{b}$ & $006 \pm 0.06_{\mathrm{c}}$ & $0.06 \pm 0.06_{\mathrm{cb}}$ & $0.00 \pm 0.00_{\mathrm{b}}$ & $0.00 \pm 0.00_{\mathrm{c}}$ & $0.00 \pm 0.00_{\mathrm{c}}$ & $0.17 \pm 0.09$ \\
\hline ES & $0.33 \pm 0.14_{b}$ & $0.61 \pm 0.18_{a}$ & $0.39 \pm 0.18_{\mathrm{ba}}$ & $0.50 \pm 0.15_{\mathrm{a}}$ & $0.28 \pm 0.11_{\mathrm{b}}$ & $0.11 \pm 0.08_{b}$ & $0.11 \pm 0.08_{\mathrm{cb}}$ & $0.06 \pm 0.06_{\mathrm{bc}}$ & $0.00 \pm 0.00$ \\
\hline MS & $0.67 \pm 0.20_{\mathrm{a}}$ & $0.61 \pm 0.20_{\mathrm{a}}$ & $0.67 \pm 0.20_{\mathrm{a}}$ & $0.67 \pm 0.21_{\mathrm{a}}$ & $0.61 \pm 0.14_{\mathrm{a}}$ & $0.61 \pm 0.14_{a}$ & $0.61 \pm 0.14_{\mathrm{a}}$ & $0.50 \pm 0.15_{\mathrm{a}}$ & $0.17 \pm 0.09$ \\
\hline PT & $0.17 \pm 0.09_{\mathrm{cb}}$ & $0.56 \pm 0.17 \mathrm{a}$ & $0.72 \pm 0.18_{\mathrm{a}}$ & $0.44 \pm 0.18_{\mathrm{ba}}$ & $0.28 \pm 0.11_{\mathrm{b}}$ & $0.22 \pm 0.13_{\mathrm{b}}$ & $0.28 \pm 0.14_{b}$ & $0.28 \pm 0.14_{\mathrm{ba}}$ & $0.39 \pm 0.18$ \\
\hline Sunflower & $0.06 \pm 0.06_{\mathrm{cb}}$ & $0.06 \pm 0.06_{b}$ & $0.11 \pm 0.11_{b}$ & $0.11 \pm 0.08_{\mathrm{bc}}$ & $0.00 \pm 0.00_{\mathrm{c}}$ & $0.06 \pm 0.06_{b}$ & $0.00 \pm 0.00_{\mathrm{c}}$ & $0.00 \pm 0.00_{\mathrm{c}}$ & $0.06 \pm 0.06$ \\
\hline $\mathrm{F}$ & 5.78 & 5.21 & 5.04 & 4.15 & 7.17 & 7.07 & 7.05 & 6.03 & 1.66 \\
\hline$P$ & 0.0001 & 0.0003 & 0.0004 & 0.0018 & 0.0001 & 0.0001 & 0.0001 & 0.0001 & 0.1505 \\
\hline \multicolumn{10}{|l|}{ c) Seeds/pods } \\
\hline Corn & $0.67 \pm 0.20_{\mathrm{a}}$ & $0.72 \pm 0.21_{\mathrm{a}}$ & $0.78 \pm 0.22_{\mathrm{a}}$ & $0.61 \pm 0.18_{\mathrm{ba}}$ & $0.67 \pm 0.20_{\mathrm{ba}}$ & $0.33 \pm 0.18_{\mathrm{cb}}$ & $0.39 \pm 0.18_{\mathrm{ab}}$ & $0.50 \pm 0.15_{\mathrm{ba}}$ & $0.61 \pm 0.14_{\mathrm{a}}$ \\
\hline ES & $0.17 \pm 0.09_{b}$ & $0.06 \pm 0.06_{b}$ & $0.22 \pm 0.10_{\mathrm{b}}$ & $0.22 \pm 0.10_{\mathrm{bc}}$ & $0.22 \pm 0.10_{\mathrm{c}}$ & $0.22 \pm 0.10_{\mathrm{cb}}$ & $0.17 \pm 0.09_{\mathrm{bc}}$ & $0.22 \pm 0.10_{\mathrm{bc}}$ & $0.17 \pm 0.09_{b}$ \\
\hline MS & $0.22 \pm 0.10_{\mathrm{b}}$ & $0.22 \pm 0.10_{\mathrm{b}}$ & $0.22 \pm 0.10_{\mathrm{b}}$ & $0.28 \pm 0.14_{\mathrm{bc}}$ & $0.28 \pm 0.14_{\mathrm{bc}}$ & $0.39 \pm 0.16_{b}$ & $0.44 \pm 0.15_{\mathrm{ab}}$ & $0.44 \pm 0.15_{\mathrm{ba}}$ & $0.06 \pm 0.06_{b}$ \\
\hline PT & $0.17 \pm 0.12_{b}$ & $0.17 \pm 0.12_{b}$ & $0.11 \pm 0.08_{b}$ & $0.11 \pm 0.08_{\mathrm{c}}$ & $0.06 \pm 0.06_{\mathrm{c}}$ & $0.11 \pm 0.08_{\mathrm{cb}}$ & $0.11 \pm 0.08_{\mathrm{bc}}$ & $0.06 \pm 0.06_{\mathrm{c}}$ & $0.06 \pm 0.06_{b}$ \\
\hline Soybean & $0.61 \pm 0.16_{\mathrm{a}}$ & $0.78 \pm 0.19_{\mathrm{a}}$ & $0.94 \pm 0.19_{\mathrm{a}}$ & $1.00 \pm 0.23_{\mathrm{a}}$ & $0.89 \pm 0.21_{\mathrm{a}}$ & $0.94 \pm 0.17_{\mathrm{a}}$ & $0.72 \pm 0.19_{\mathrm{a}}$ & $0.78 \pm 0.17_{\mathrm{a}}$ & $0.78 \pm 0.15_{\mathrm{a}}$ \\
\hline Sunflower & $0.00 \pm 0.00_{\mathrm{b}}$ & $0.00 \pm 0.00_{\mathrm{b}}$ & $0.00 \pm 0.00_{\mathrm{b}}$ & $0.00 \pm 0.00_{\mathrm{c}}$ & $0.00 \pm 0.00_{\mathrm{c}}$ & $0.00 \pm 0.00_{\mathrm{c}}$ & $0.00 \pm 0.00_{\mathrm{c}}$ & $0.00 \pm 0.00_{c}$ & $0.00 \pm 0.00_{\mathrm{b}}$ \\
\hline $\mathrm{F}$ & 4.38 & 6.36 & 8.01 & 6.88 & 6.44 & 6.28 & 3.94 & 6.16 & 11.35 \\
\hline $\mathrm{P}$ & 0.0012 & 0.0001 & 0.0001 & 0.0001 & 0.0001 & 0.0001 & 0.0026 & 0.0001 & 0.0001 \\
\hline
\end{tabular}

Abbreviations: ES=Early Scarlet cowpea; MS=Mississippi Silver cowpea; $\mathrm{PT}=$ Princess Tree; $\mathrm{P}=\mathrm{P}$-value; F=F-value.

$\dagger$ Means followed by the same letter within the same column are not significantly different ( $\mathrm{df}=5,102 ; \mathrm{P}>0.05$; Tukey's method).

\subsubsection{Different Growth Stages from the Same Plant}

Halyomorpha halys was exposed to all three growth stages (leaves, flowers and pods) for each test plant in a MCAT to determine its preference. Our results show that when $H$. halys adults were exposed to all three growth stages (leaves, flowers and pods) of Early Scarlet cowpea there were significant differences in its distribution on the different growth stages (Table 4a). More H. halys were recorded on Early Scarlet flowers and pods than on its leaves (Table 4a). Similarly, result from Table $4 \mathrm{~b}$ shows that most $H$. halys were recorded on flowers and pods of Mississippi Silver cowpea. From Table 4c, our result shows that at each time interval more $H$. halys were recorded on soybean pods than on the flowers and leaves. Halyomorpha halys distribution on the leaves, flowers and corn kernel (seed) was significantly different at each time interval (Table 4d). At each time interval, corn kernel was the most preferred compared to the leaves and flowers (Table 4d). H. halys distribution on the different sunflower growth parts was only significant at 3, 5 and 24 hours after release of $H$. halys in test arena (Table 4e). During these times, the flowers were preferred over the leaves and seeds. There was no effect of time on the selection of $H$. halys to the different growth stages for each host plant.

Table 4. Mean number $( \pm S E)$ of $H$. halys observed on the different growth stages (leaf, flower and kernels/seeds/pods) from the same test plant over time.

\begin{tabular}{|c|c|c|c|c|c|c|c|}
\hline \multirow{2}{*}{ Test plants } & \multicolumn{7}{|c|}{ Time (hours) } \\
\hline & 1 & 2 & 3 & 4 & 5 & 6 & 24 \\
\hline \multicolumn{8}{|l|}{ (a) ES } \\
\hline Leaves & $0.00 \pm 0.00_{\mathrm{b}}$ & $0.00 \pm 0.00_{\mathrm{b}}$ & $0.22 \pm 0.14_{b}$ & $0.33 \pm 0.16$ & $0.11 \pm 0.10_{\mathrm{b}}$ & $0.00 \pm 0.00_{\mathrm{b}}$ & $0.11 \pm 0.10_{\mathrm{b}}$ \\
\hline Flowers & $0.78 \pm 0.26_{a}$ & $0.67 \pm 0.22_{\mathrm{a}}$ & $1.22 \pm 0.26_{\mathrm{a}}$ & $0.78 \pm 0.31$ & $0.89 \pm 0.25_{\mathrm{a}}$ & $0.89 \pm 0.29_{\mathrm{a}}$ & $0.22 \pm 0.14_{b}$ \\
\hline Pods & $0.33 \pm 0.16_{\mathrm{ab}}$ & $0.78 \pm 0.26_{a}$ & $0.89 \pm 0.33_{\mathrm{ab}}$ & $1.22 \pm 0.34$ & $1.56 \pm 0.36_{\mathrm{a}}$ & $1.67 \pm 0.38_{\mathrm{a}}$ & $1.67 \pm 0.35_{\mathrm{a}}$ \\
\hline $\mathrm{F}$ & 4.39 & 4.37 & 3.75 & 1.99 & 7.28 & 8.53 & 12.55 \\
\hline $\begin{array}{l}\mathrm{P} \\
\text { (b) MS }\end{array}$ & 0.0237 & 0.0241 & 0.0382 & 0.1583 & 0.0034 & 0.0016 & 0.0002 \\
\hline Leaves & $0.00 \pm 0.00$ & $0.00 \pm 0.00$ & $0.00 \pm 0.00_{b}$ & $0.00 \pm 0.00_{\mathrm{b}}$ & $0.00 \pm 0.00_{b}$ & $0.00 \pm 0.00_{\mathrm{b}}$ & $0.00 \pm 0.00_{\mathrm{b}}$ \\
\hline Flowers & $0.44 \pm 0.23$ & $0.78 \pm 0.31$ & $1.00 \pm 0.31_{\mathrm{a}}$ & $0.78 \pm 0.31_{\mathrm{a}}$ & $0.89 \pm 0.25_{\mathrm{a}}$ & $0.56 \pm 0.17_{\mathrm{ab}}$ & $0.00 \pm 0.00_{\mathrm{b}}$ \\
\hline Pods & $0.56 \pm 0.28$ & $0.78 \pm 0.34$ & $1.33 \pm 0.38_{\mathrm{a}}$ & $1.00 \pm 0.35_{\mathrm{a}}$ & $1.11 \pm 0.37_{\mathrm{a}}$ & $1.33 \pm 0.47_{\mathrm{a}}$ & $1.44 \pm 0.42_{\mathrm{a}}$ \\
\hline
\end{tabular}




\begin{tabular}{|c|c|c|c|c|c|c|c|}
\hline \multirow{2}{*}{ Test plants } & \multicolumn{7}{|c|}{ Time (hours) } \\
\hline & 1 & 2 & 3 & 4 & 5 & 6 & 24 \\
\hline $\mathrm{F}$ & 1.86 & 3.06 & 6.1 & 3.98 & 5.20 & 5.00 & 12.73 \\
\hline $\mathrm{P}$ & 0.1771 & 0.0653 & 0.0072 & 0.0323 & 0.0133 & 0.0154 & 0.0002 \\
\hline \multicolumn{8}{|l|}{ (c) Soybean } \\
\hline Leaves & $0.11 \pm 0.10$ & $0.00 \pm 0.00_{\mathrm{b}}$ & $0.11 \pm 0.10_{\mathrm{b}}$ & $0.00 \pm 0.00_{\mathrm{b}}$ & $0.11 \pm 0.10_{\mathrm{b}}$ & $0.00 \pm 0.00_{\mathrm{b}}$ & $0.22 \pm 0.14_{\mathrm{b}}$ \\
\hline Flowers & $0.22 \pm 0.14$ & $0.22 \pm 0.14_{b}$ & $0.56 \pm 0.23_{\mathrm{ab}}$ & $0.44 \pm 0.17_{\mathrm{ab}}$ & $0.44 \pm 0.23_{b}$ & $0.11 \pm 0.10_{\mathrm{b}}$ & $0.22 \pm 0.14 \mathrm{~b}$ \\
\hline Pods & $0.56 \pm 0.28$ & $1.00 \pm 0.42_{\mathrm{a}}$ & $0.89 \pm 0.25_{\mathrm{a}}$ & $1.22 \pm 0.47_{\mathrm{a}}$ & $1.56 \pm 0.45_{\mathrm{a}}$ & $2.00 \pm 0.42_{\mathrm{a}}$ & $1.11 \pm 0.40_{\mathrm{a}}$ \\
\hline $\mathrm{P}$ & 0.3319 & 0.0223 & 0.0489 & 0.0225 & 0.0096 & 0.0001 & 0.0357 \\
\hline \multicolumn{8}{|l|}{ (d) Corn } \\
\hline Leaves & $0.00 \pm 0.00_{\mathrm{b}}$ & $0.00 \pm 0.00_{\mathrm{b}}$ & $0.11 \pm 0.10_{\mathrm{b}}$ & $0.11 \pm 0.10_{\mathrm{b}}$ & $0.11 \pm 0.10_{\mathrm{b}}$ & $0.00 \pm 0.00_{\mathrm{b}}$ & $0.22 \pm 0.14_{\mathrm{b}}$ \\
\hline Flowers & $0.00 \pm 0.00_{\mathrm{b}}$ & $0.00 \pm 0.00_{\mathrm{b}}$ & $0.00 \pm 0.00_{\mathrm{b}}$ & $0.11 \pm 0.10_{\mathrm{b}}$ & $0.11 \pm 0.10_{\mathrm{b}}$ & $0.11 \pm 0.10_{\mathrm{b}}$ & $0.00 \pm 0.00_{\mathrm{b}}$ \\
\hline Kernels & $0.67 \pm 0.27_{\mathrm{a}}$ & $1.33 \pm 0.47_{\mathrm{a}}$ & $1.56 \pm 0.42_{\mathrm{a}}$ & $1.67 \pm 0.35_{\mathrm{a}}$ & $1.67 \pm 0.27 \mathrm{a}$ & $1.33 \pm 0.42_{\mathrm{a}}$ & $0.89 \pm 0.19_{\mathrm{a}}$ \\
\hline $\mathrm{F}$ & 5.70 & 8.17 & 11.2 & 14.82 & 23.43 & 9.38 & 11.02 \\
\hline $\mathrm{P}$ & 0.0094 & 0.0020 & 0.0004 & 0.0001 & 0.0001 & 0.0010 & 0.0004 \\
\hline Leaves & $0.00 \pm 0.00$ & $0.00 \pm 0.00$ & $0.11 \pm 0.10_{\mathrm{b}}$ & $0.33 \pm 0.22$ & $0.33 \pm 0.22_{\mathrm{ab}}$ & $0.00 \pm 0.00$ & $0.22 \pm 0.14_{b}$ \\
\hline Flowers & $0.11 \pm 0.10$ & $0.33 \pm 0.16$ & $1.00 \pm 0.27_{\mathrm{a}}$ & $0.67 \pm 0.22$ & $0.89 \pm 0.29 \mathrm{a}$ & $0.00 \pm 0.00$ & $0.89 \pm 0.25_{\mathrm{a}}$ \\
\hline Pods & $0.00 \pm 0.00$ & $0.11 \pm 0.10$ & $0.00 \pm 0.00_{\mathrm{b}}$ & $0.00 \pm 0.00$ & $0.00 \pm 0.00_{\mathrm{b}}$ & $0.00 \pm 0.00$ & $0.00 \pm 0.00_{b}$ \\
\hline $\mathrm{F}$ & 1.00 & 2.15 & 9.86 & 3.40 & 4.20 & & 7.44 \\
\hline $\mathrm{P}$ & 0.3827 & 0.1379 & 0.0007 & 0.0501 & 0.0273 & & 0.0031 \\
\hline
\end{tabular}

Abbreviations: $\mathrm{ES}=$ Early Scarlet cowpea; $\mathrm{MS}=$ Mississippi Silver cowpea; $\mathrm{P}=\mathrm{P}$-value; $\mathrm{F}=\mathrm{F}$-value.

$\dagger$ Means followed by the same letter within the same column are not significantly different ( $\mathrm{P}>0.05$; Tukey's method).

\section{Discussion}

Halyomorpha halys is a highly polyphagous insect with an extensive host plant range that includes fruits, ornamental plants and vegetable crops. Among these immense host plants, $H$. halys, like any phytophagous insect, exhibits some degree of selectivity of host plant for feeding and reproduction [27]. According to $[28,29]$, insects would choose to oviposit on those host plants that maximize the fitness of their offspring, and they would prefer to feed on those that provide the best adult performance. From laboratory bioassays, when $H$. halys was given a choice of leaves only from six host plants (corn, Early Scarlet cowpea, Mississippi Silver cowpea, soybean, sunflower and princess tree) in both Dual Choice Arena Test (DCAT) and Multiple Choice Arena Test (MCAT), our results show that more $H$. halys were recorded on princess tree leaves compared to leaves from the other host plants (Figure 1a, Table $3 a)$. These findings are in agreement with field reports indicating that princess tree was among the initial host plants of adult $H$. halys after emerging from over wintering sites in April and May. This is where females deposited egg masses on the underside of leaves [20, 26]. After oviposition, H. halys remained on princess tree as indicated in a host plant survey by [23] which reported that during the summer months (July to September), princess tree still appeared highly preferred by $H$. halys. Even though our current study did not evaluate developmental studies, previous work reported $>80 \%$ survival of $H$. halys on princess tree leaf (as a single food substrate) at each nymph stage however, this was accompanied by a longer developmental time and decreased weight [20]. From our findings the high preference for princess tree leaves by $H$. halys could be an indicator that this tree is one of the early host plant used by $H$. halys for oviposition. In addition, this preference could be mediated by morphological and biochemical composition of the plant or a combination of both. For example, results from [30] showed that the preference of $H$. halys for bell peppers of a particular color and stage of maturity may be a result of the differences in protein concentration.

Generally, during the period when H. halys emerges from overwintering sites (April and May) [20], there are fewer vegetable crops in the field and if present, these crops are usually in the seedling growth stage which is hardly attacked by $H$. halys. When given a choice between leaves of princess tree and leaves from the other host plant in our laboratory bioassay, $H$. halys showed preference for princess tree leaves over the other leaves. However, the scenario was different in the distribution and in the number of $H$. halys on princess tree leaves in a choice and no choice tests. In a choice test (DCAT X PT), the number of $H$. halys recorded on princess tree leaves was less compared to the number in a no choice test (PT-PT) (Table 2a). This is not surprising because in the presence of other host plants $H$. halys dispersed to leaves of the other host plants. This implies that when $H$. halys was given a choice of leaves of other host plant in this case corn, soybean, sunflower, cowpea (Mississippi Silver and Early Scarlet), some were attracted to the other host plants resulting in a reduction in the density or number of $H$. halys recorded on any one particular crop. However, this behavior did not change the high preference $H$. halys has for princess tree leaves over leaves of the other host plants as shown by the Preference Index (PI) value (Figure 1a, Table 1a).

Despite their expansive host-range, plant use by pentatomids changes with host maturity and phenology [31]. And as the plants mature and develop dry pods and seeds, they become less attractive, and stink bugs move to other preferred plants [32]. From our findings, the reproductive structures were more preferred than the leaves (Figure 1, Tables 3 and 4). In both DCAT and MCAT, flowers of Mississippi Silver and Early Scarlet cowpea, and pods of Mississippi Silver and Early Scarlet cowpea and soybean and 
corn kernel were more preferred than princess tree leaves. This behavior of $H$. halys observed in our study conform to other reports. For example, highest density of $H$. Halys were observed on developing corn kernel and soybean pods in the field [33-36] and in other hemiptera such as Nezara viridula (Hemiptera: Pentatomidae), where laboratory studies showed a stronger attraction to mature soybean pods [14]. Similarly, [37] reported the highest infestation of the legume pod borer, Maruca testulalis (Lepidoptera: Crambidae), in cowpea flowers compared to the other growth stages. Difference in the quality of food resources from the different plant parts (leaves, flowers, and fruits) has been shown to play an important role in the performance of phytophagous insects [38]. According to [20], H. halys switched from one host plant to another on the field probably in search of the most nutritious food source. Selection of the proper food source for growth and development is crucial for the development and establishment of insects. Therefore, these stages which represent the period of availability of both proteins and carbohydrates materials in high proportion for the insects [34] were more preferred. In addition, the efficacy of a trap crop seems to depend on the phenology of the trap crop and the cash crop plants. Overall, the reproductive components (flowers and pods) of Mississippi Silver and Early Scarlet cowpeas were highly preferred by H. halys than the other host plants.

\section{Conclusions}

Even though $H$. halys is reported to attack over a hundred other host plants than those tested in this study, our results demonstrate its preference for Mississippi Silver (MS) and Early Scarlet (ES) flowers and pod and suggest that these preferred cowpea varieties may be used as a trap crop in an integrated management program for this pest. However, before being recommended, these alternatives must be further investigated under field conditions.

\section{Acknowledgements}

We thank members of the Integrated Pest Management Laboratory at North Carolina A\&T State University for their assistance. This research was funded by the United States Department of Agriculture and the National Institute of Food and Agriculture (USDA-NIFA) Evans Allen Program, Grant No. NC. X-287-5-15-170-1.

\section{References}

[1] Stopbmsb.org Management of brown marmorated stink bug in US specialty crops http://www.stopbmsb.org/where-is-bmsb/ (accessed April 20).

[2] Kiritani, K. (2006). Predicting impacts of global warming on population dynamics and distribution of arthropods in Japan. Population Ecology, 48 (1): 5-12.

[3] Fogain, R and Graff, S (2011). First records of the invasive pest,
Halyomorpha halys (Hemiptera: Pentatomidae), in Ontario and Quebec. Journal of the Entomological Society of Ontario, 142: $45-48$.

[4] Wermelinger, B. Wyniger, D and Forster, B. (2008). First records of an invasive bug in Europe: Halyomorpha halys Stal (Heteroptera: Pentatomidae), a new pest on woody ornamentals and fruit trees? Mitteilungen der Schweizerischen Entomologischen Gesellschaft, 81: 1-8.

[5] Arnold, K (2009). Halyomorpha halys (Stål 1855) a stink bug species newly detected among the European fauna (Insecta: Heteroptera, Pentatomidae, Pentatominae, Cappaeini). Eine fur die europaische Fauna neu nachgewiesene Wanzenart. Mitteilungen des Thüringer Entomologenverbandes, 16: 1-19.

[6] Callot, H. Brua. (2013). Halyomorpha halys (Stål, 1855), la Punaise diabolique, nouvelle espèce pour la faune de France (Heteroptera: Pentatomidae). The diabolic bug, a new fauna of France. L'Entomologiste, 69: 69-71.

[7] Heckmann, R and Erster Nachweis von (2012). Halyomorpha halys (Stal, 1855) (Heteroptera: Pentatomidae) für Deutschland. Heteropteron 36: 17-18.

[8] Pansa, M. G. Asteggiano, L. Costamagna, C. Vittone, G and Tavella (2013). First discovery of Halyomorpha halys in peach orchards in Piedmont. [Primo ritrovamento di Halyomorpha halys nei pescheti piemontesi.] Informatore Agrario 69: 60-61.

[9] Milonas, P and Partsinevelos, G. (2014). First report of brown marmorated stink bug Halyomorpha halys Stål (Hemiptera: Pentatomidae) in Greece. EPPO Bulletin 44 (2): 183-186.

[10] Rabitsch, W and Friebe (2015). From the west and from the east? First records of Halyomorpha halys (Stål, 1855) (Hemiptera: Heteroptera: Pentatomidae) in Vorarlberg and Vienna, Austria. Beiträge zur Entomofaunistik, 16: 126-129.

[11] Vetek, G. Papp, V. Haltrich, A and Redei (2014). First record of the brown marmorated stink bug, Halyomorpha halys Hemiptera: Heteroptera: Pentatomidae), in Hungary, with description of the genitalia of both sexes. Zootaxa, 3780: 194-200.

[12] Macavei, L. Bâe, R. Oltean, I. Florian, T. Mircea Ioan, V. Costi, $\mathrm{E}$ and Maistrello (2015). First detection of Halyomorpha halys Stål, a new invasive species with a high potential of damage on agricultural crops in Romania. 58: 105-108.

[13] Leskey, T. Short, B. Butler, B and Wright (2012). Impact of the Invasive brown marmorated stink bug, Halyomorpha halys (Stål), in mid-atlantic tree fruit orchards in the United States: Case studies of commercial management. Psyche, 2012: 1-14.

[14] Molina, G. A and Trumper (2012). Selection of soybean pods by the stink bugs, Nezara viridula and Piezodorus guildinii. Journal of Insect Science, 12.

[15] United States Apple Association (USAA) Brown Marmorated Stink Bug Causes \$37 Million in Losses to Mid-Atlantic Apple Growers.

http://www.growingproduce.com/fruits/apples-pears/brown-m armorated-stink-bug-causes-37-million-in-losses-to-mid-atlant ic-apple-growers/.

[16] Leskey, T. Hamilton, G. Nielsen, A. Polk, D. Rodriguez-Saona, C.; Bergh, J. et al. (2012). Pest Status of the Brown Marmorated Stink Bug, Halyomorpha Halys in the USA. Outlooks on Pest Management, 23: 218-226. 
[17] Shelton, A. M and Badenes-Perez (2006) Concepts and applications of trap cropping in pest management. Annal Review of Entomology, 51: 285-308.

[18] Hokkanen, H. M. T (1991) Trap Cropping in Pest Management. Annual Review of Entomology, 36 (1): 119-138.

[19] Parker, J. E. Snyder, W. E. Hamilton, G. C. and Rodriguez-Saona (2013). Companion planting and insect pest control. Weed and Pest Control - Conventional and New Challenges, 1-30.

[20] Dingha, B. N and Jackai, L. E. N (2016). Laboratory rearing of the brown marmorated stink bug (Hemiptera: Pentatomidae) and the impact of single and combination of food substrates on development and survival. Canadian Entomologist, 149 (1): 104-117.

[21] Kogan, M and Goeden (1970). Host-Plant range of Lema trilineata daturaphila (Coleoptera - Chrysomelidae). Annals of Entomoogical Society of America, 63 (4), 1175-1180.

[22] Lin, H. C. Kogan, M and Fischer (1990). Induced resistance in soybean to the Mexican Bean Beetle (Coleoptera, Coccinellidae) - Comparisons of inducing factors. Environmental Entomology, 19 (6): 1852-1857.

[23] Bakken, A. J. Schoof, S. C. Bickerton, M. Kamminga, K. L. Jenrette, J. C.; Malone, S. Abney, M. A et al. (2015). Occurrence of brown marmorated stink bug (Hemiptera: Pentatomidae) on wild hosts in non-managed woodlands and soybean fields in North Carolina and Virginia. Environmental Entomology, 44 (4): 1011-1021.

[24] Aigner, B. L. Kuhar, T. P. Herbert, D. A. Brewster, C. C. Hogue, J. W and Aigner (2017). Relationship of brown marmorated stink bug, Halyomorpha halys, infestations in tree borders to subsequent patterns of movement into soybean fields Journal of Econnmic Entomology, 110 (2): 487-490.

[25] Dingha, B. N and Jackai, L. E. N (2014). Laboratory rearing and field monitoring of the brown marmorated stink bug (Halyomorpha halys) in the Greensboro area of North Carolina. In: Abstracts ESA southeastern branch meeting, Greenville, NC, p 56.

[26] Nielsen, A. L and Hamilton (2009). Life history of the invasive species Halyomorpha halys (Hemiptera: Pentatomidae) in Northeastern United States. Annals of Entomological Society of America, 102 (4): 608-616.

[27] Bernays, E. A and Chapman (1994). Host-plant selection by phytophagous insects. Chapman \& Hall: New York; London, p xiii, $312 \mathrm{p}$.
[28] Jaenike, J (1978). Optimal Oviposition Behavior in Phytophagous Insects. Theor Population Biology, 14 (3): 350-356.

[29] Stephens, D. W and Krebs (1986). Foraging theory. Princeton University Press.

[30] Mensah-Bonsu, M. Dingha, B. N. Jackai, L. E. N. Adjei-Fremah, S and Worku (2020). Evaluation of preference of brown marmorated stink bug, Halyomorpha halys (Stål) for different colour bell peppers and the role of plant protein. Arthropod-Plant Interact, 14: 363-372.

[31] Bundy, C. S and McPherson (2000). Dynamics and seasonal abundance of stink bugs (Heteroptera: Pentatomidae) in a cotton-soybean ecosystem. Journal of Economic Entomology, 93 (3): 697-706.

[32] McPherson, J. E and McPherson (2000). Stink bugs of economic importance in America North of Mexico. p 1-254.

[33] Nielsen, A. L. Hamilton, G. C and Shearer (2011). Seasonal phenology and monitoring of the non-native Halyomorpha halys (Hemiptera: Pentatomidae) in soybean. Environmental Entomology, 40 (2): 231-238.

[34] Venugopal, P. D. Dively, G. P and Lamp (2015). Spatiotemporal dynamics of the invasive Halyomorpha halys (Hemiptera: Pentatomidae) in and between adjacent corn and soybean fields. Journal of Economic Entomology 108 (5): 2231-41.

[35] Koch, R. L and Rich (2015). Stink bug (Hemiptera: Heteroptera: Pentatomidae) feeding and phenology on early-maturing soybean in Minnesota. Journal of Economic Entomology, 108 (5): 2335-2343.

[36] Zobel, E. S. Hooks, C. R. R and Dively (2016). Seasonal abundance, host suitability, and feeding injury of the brown marmorated stink bug, Halyomorpha halys (Heteroptera: Penatomidae), in selected vegetables. Journal of Economic Entomology, 109 (3): 1289-1302.

[37] Jackai, L. E. N (1981). Relationship between cowpea crop phenology and field infestation by the legume pod borer, Maruca testulalis (Lepidoptera, Pyralidae). Annals of Entomological Society of America, 74 (4), 402-408.

[38] Scheirs, J. Jordaens, K and De Bruyn (2005). Have genetic trade-offs in host use been overlooked in arthropods? Evolutionary Ecology, 19: 551-561. 